\title{
ADOLESZENTE WEIBLICHKEIT IM GRENZREGIME: FREMD- UND SELBSTPOSITIONIERUNGEN JUNGER AUS SOMALIA GEFLÜCHTETER FRAUEN* AUF MALTA
}

\author{
Laura Otto und Margrit E. Kaufmann
}

Goethe-Universität Frankfurt am Main, Institut für Kulturanthropologie und Europäische Ethnologie

E-Mail: otto@em.uni-frankfurt.de

URL: https://www.uni-frankfurt.de/77690794/Dr_LLaura_Otto

Universität Bremen, Institut für Ethnologie und Kulturwissenschaft

E-Mail: mkaufm@uni-bremen.de

URL: https://www.uni-bremen.de/kultur/personen/lektorinnen/margrit-e-kaufmann

Zitationsvorschlag:

Otto, Laura/Kaufmann, Margrit E. (2021): Adoleszente Weiblichkeit im Grenzregime: Fremd- und Selbstpositionierungen junger aus Somalia geflüchteter Frauen* auf Malta. In: Gesellschaft - Individuum - Sozialisation (GISo). Zeitschrift für Sozialisationsforschung, 2 (1). DOI: 10.26043/GISo.2021.1.2

Link zum Artikel:

https://doi.org/10.26043/G/So.2021.1.2 


\title{
ADOLESZENTE WEIBLICHKEIT IM GRENZREGIME: FREMD- UND SELBSTPOSITIONIERUNGEN JUNGER AUS SOMALIA GEFLÜCHTETER FRAUEN* AUF MALTA
}

\author{
Laura Otto und Margrit E. Kaufmann
}

\begin{abstract}
Die Erfahrungen von Frauen* und Mädchen* sind in den Debatten um Flucht und Migration unterbeleuchtet. Der Beitrag greift diese Lücke auf, indem er nach Perspektiven und Erfahrungen junger, weiblich gelesener Geflüchteter somalischer Herkunft fragt, die in der Europäischen Union auf Malta ankamen. Anhand von gemeinsam gelesenen und interpretierten ethnographisch erhobenen Materialien von Laura Otto zeigen wir im gemeinsam verfassten Text, wie auf Malta mit Weiblichkeit im Fluchtkontext umgegangen wird. Dabei befassen wir uns mit den Selbst- und Fremdpositionierungen junger geflüchteter Frauen*. Als drei zentrale Narrative und Umgangsweisen heben wir heraus, wie diese (nicht) über Flucht und Gewalt sprechen, wie sie mit dem (nicht vorhandenen) Schutzstatus umgehen und welche Erwartungen von außen an sie gestellt werden. In den ethnographischen Beschreibungen deutet sich Weiblichkeit mit anderen sozial konstruierten Kategorien wie Alter und Herkunft als verwoben an. Entsprechend zielt der Beitrag auf intersektionelle Leseweisen ethnographischer Forschungsmaterialien zu Weiblichkeit im Grenzregime und auf deren Reflexion, gerade auch mittels gemeinsamer Interpretation der Schreibenden.
\end{abstract}

Keywords: Flucht, Frauen, Mädchen, Intersektionalität, ethnographische Methoden, Zuschreibungen, Grenzregime

\section{PERSPEKTIVEN UND ERFAHRUNGEN JUN- GER, WEIBLICH GELESENER GEFLÜCHTETER IM FOKUS}

Frauen*1 machen zwar weltweit rund die Hälfte aller sich auf der Flucht befindenden Menschen aus; in Europa wird jedoch nur rund ein Drittel aller Asylanträge von Frauen* gestellt (Hanevinkel 2018). Die öffentliche und akademische Debatte um Flucht_Migration ist derzeit primär vom Bild (junger) männlicher* Geflüchteter geprägt. Oft werden sie als problematisch und hyper-maskulin dargestellt, aber auch als diejenigen, denen die Flucht am ehesten gelingt (Spindler 2006; Huxel 2014, 11; Otto/Kaufmann 2018). Die spezifischen Geschichten und Lebenssituationen von geflüchteten Mädchen* und Frauen*, die es bis nach Europa schaffen, erfahren vergleichsweise weniger Beachtung und sind auch in der Forschung unterrepräsentiert (Çalışkann 2018, 12).

Um dem entgegenzuwirken, rückt dieser Beitrag weiblich gelesene junge Geflüchtete ins Zentrum. Während sich also das vorliegende Heft der Frage widmet, wie Kindheit und/oder Adoleszenz unter den spezifischen Bedingungen von Flucht_Migration erlebt und gestaltet werden, fokussieren wir diese wichtige Frage im Hinblick auf die Herstellung von und den Umgang mit adoleszenter Weiblichkeit im europäischen Grenzregime. ${ }^{2}$ Am Beispiel wird sich zeigen, dass Geschlecht, Alter und Herkunft im

\footnotetext{
${ }^{1}$ Wir verwenden den Genderstar um neben männlichen und weiblichen auch weitere Geschlechter und Geschlechtsidentitäten mitzudenken und typografisch sichtbar zu machen (Goel 2020). Bei denjenigen Personen, die in diesem Artikel zitiert werden und bei denen wir über die Selbstpositionierung informiert sind, verzichten wir auf den * und nutzen in diesen Fällen die jeweilige Selbstbezeichnung (er/sie).

2 Unter Grenzregime verstehen wir in diesem Beitrag ein heterogenes Ensemble aus menschlichen (z. B. Politiker*innen, Geflüchtete, NGO-Mitarbeiter*innen) und mehr-als-menschlichen Akteur*innen (z. B. Visa-Regularien, Checkpoints, Lager), die an komplexen Inklusions- und Exklusionsprozessen beteiligt sind. Grenze wird hier nicht als feststehend verstanden, sondern als dynamisch, relational und prozessual (Opitz 2011; Hess/Tsianos 2010).
} 
Flucht_Migrationskontext eng miteinander verwoben sind und dass für das Thematisieren von Kindheit und Adoleszenz den sozialen Kategorien Geschlecht und Herkunft wichtige Bedeutungen zukommen. Konkret befassen wir uns mit der Situation junger geflüchteter Frauen* somalischer Herkunft, die nach ihrer Ankunft auf Malta entweder als unbegleitete minderjährige Geflüchtete $\left(\cup A M^{3}\right)$ oder als junge Erwachsene eingeteilt wurden. Wir fokussieren den situativen Umgang mit gelesener adoleszenter Weiblichkeit im Fluchtkontext in Form von Selbst- und Fremdzuschreibungen aus einer relationalen Perspektive. Praktiken des Umgangs mit Weiblichkeit üben im Beispiel der Forschung sowohl die jungen geflüchteten Frauen* selbst aus als auch die sie Betreuenden, Verwaltenden sowie (junge) geflüchtete Männer* und wir Forscherinnen. Diesem Umgang gehen wir entlang von drei zentralen Narrativen nach: dem (Nicht-)Sprechen über Flucht und Gewalt, dem Umgang mit dem (nicht vorhandenen) Schutzstatus sowie den von außen gestellten Erwartungen an junge Frauen* mit Reglementierungen als Folge.

Entgegen einem oftmals essentialisierenden und/oder paternalistischen Umgang mit jungen Frauen* in Fluchtkontexten versuchen wir Dynamiken der Fremd- und Selbstzuschreibung mittels Ethnographie sichtbar zu machen, ohne gängige Verallgemeinerungen etablierter Konzepte unhinterfragt zu reproduzieren. Zur Anregung eines reflektierenden Umgangs mit dieser Herausforderung lesen und interpretieren wir ähnlich wie bereits bezüglich junger männlicher* Geflüchteter (Otto/Kaufmann 2018, 2020) - gemeinsam ethnographische Materialausschnitte von Laura Otto.

Der Beitrag ist entsprechend wie folgt aufgebaut: Zunächst geben wir einen Einblick in den Forschungsstand zu Mädchen* und Frauen* im Fluchtkontext, in dem wir den Text verorten. Anschließend erläutern wir die angewendeten Forschungsmethoden, nehmen methodologisch Bezug auf Verbindungen von Ethnographie und Intersektionalität und stellen unseren Ansatz einer intersektionellen Leseweise vor. Im Anschluss verdeutlichen wir anhand empirischer
Materialien, wie mit Weiblichkeit im Fluchtkontext auf Malta umgegangen wird. Wir schließen den Beitrag mit Erkenntnissen zu Selbst- und Fremdpositionierungen von jungen geflüchteten Frauen* und einer Diskussion zum methodischen Vorgehen ab.

\subsection{Forschungsstand zu Mädchen*/Frauen* und Flucht}

Die Flucht_Migrationsforschung ist von einem „male-centered paradigm“ (Callamard 2002) geprägt. Das trifft sowohl auf internationale Forschungen zu als auch auf die deutschsprachige Flucht_Migrationsforschung, in der immer wieder Perspektiven und Erfahrungen geflüchteter Männer* zentral gesetzt werden (Spindler 2006; Tunç 2018; Scheibelhofer 2019). Dieser „malebias", obgleich weltweit immer mehr Frauen* auf der Flucht sind, trägt dazu bei, dass deren Perspektiven, Praktiken und spezifische Lebensumstände in der Forschung unterrepräsentiert sind (Lutz/Amelina 2021; Kholi 2007; Deacon/Sullivan 2009). Eileen Pittaway und Linda Bartolomei $(2001,22)$ sprechen von einer "gendered nature of the refugee experience" und kritisieren vor allem Regelwerke und Gesetze dafür, frauen*spezifische Fluchtumstände weitgehend zu ignorieren. Alison Gerard und Sharon Pickering (2013) diskutieren die gegenderten Dynamiken von Bordering-Prozessen entlang der EUAußengrenze. Untersucht wurde dieses Phänomen vor allem in Bezug auf den Zugang zum Flüchtlingsstatus in Ländern des Globalen Nordens. Einen weiteren Fokus bilden die Erfahrungen von Frauen* in Camps (Spijkerboer 2000; Bhabha 2004; Pickering 2011).

Detailliert arbeiten Ravi Kholi und Mervi Kaukko (2018) heraus, wie junge geflüchtete Frauen* und Mädchen* mit dem Warten auf ihre Asylstatusentscheidung umgehen und dabei wichtige soziale Kontakte knüpfen. Ala Sirriyeh (2013) fokussiert die Praktiken und Perspektiven junger geflüchteter Frauen* in England, hält der gängigen Dichotomie von „victims or villains“ einen differenzierteren Blick entgegen und arbeitet heraus, wie unterschiedlich die jungen Frauen* „home" verstehen und in England praktizieren.

\footnotetext{
${ }^{3}$ UAM steht für , unaccompanied minor'. Mit dieser englischen Bezeichnung wurden die jungen Geflüchteten auf Malta betitelt.
} 
Marianne Vervliet et al. (2013) betrachten aus einer intersektionellen Perspektive, wie sich die Kategorien „refugee“, „mother“, „unaccompanied“ und „adolescent“ miteinander verweben.

Diese Forschungen zeigen, dass mit Frauen* und Mädchen* im Fluchtkontext anders umgegangen wird als mit Jungen* und Männern* und dass auch sie selbst einen anderen Umgang mit ihren Situationen entwickeln. Um diesen vielfältigen Umgangsweisen nachspüren zu können und überhaupt einen analytischen Zugang zu den komplexen Selbst- und Fremdpositionierungen zu erlangen, greifen wir ethnographische Forschungsmaterialien von Laura Otto (2020) auf. Durch die Betreuung des Forschungsprojekts durch Margrit Kaufmann sind beide mit der Forschung, deren Materialien sie für diesen Text gemeinsamen Deutungsrunden unterzogen haben, vertraut - die eine aus der Innensicht der Ethnographin, die andere aus der Außensicht der Begleitung mit Fokus auf Intersektionalitätsforschung (Kaufmann 2019). Der folgende Abschnitt skizziert die ethnographischen Grundlagen.

\subsection{Ethnographischer Zugang, Forschungsteil- nehmerinnen* und Forscherin}

Das Material, auf das wir Bezug nehmen, ist Teil eines langfristigen ethnographischen Forschungsprojektes (2013-2018) von Laura Otto, welches sich mit Prozessen der Altersaushandlung im EU-Grenzregime im maltesischen Kontext befasst. ${ }^{4}$ Der Forschungszugang zu einer staatlichen Unterbringungseinrichtung für UAM führte sie an einen Ort, der von transkulturellen Begegnungen geprägt war. Hier konnte sie im Feld Sozialer Arbeit zum Gegenstandsbereich von Flucht forschen, einem Bereich, in dem unter machtvollen Bedingungen mit sozialer Vielfalt umgegangen wird. In ethnographischer Tradition folgte sie den Verbindungen, die sich während der Forschung ergaben. Das erhobene Material besteht aus narrativen Interviews mit geflüchteten und nicht-geflüchteten Akteur*innen, informellen Gesprächen und teilnehmenden Beobachtungen. 48 junge Geflüchtete, die als UAM kategorisiert wurden, und 17 Geflüchtete, die als nicht-minderjährig galten, wurden ebenso Teil der Forschung wie 27 nicht-geflüchtete Akteur*innen, unter anderem Sozialarbeitende, Ministeriumsangestellte und NGO-Mitarbeitende. Gesetzestexte, NGO-Berichte sowie Zeitungsartikel ergänzen den Materialkorpus.

Um Beziehungen zu den jungen Menschen mit Flucht_Migrationserfahrung aufzubauen, verbrachte Laura Otto 2013 über sechs Monate nahezu jeden Tag mit den jungen Geflüchteten. Zunächst trafen sie sich in der Unterbringungseinrichtung. Später, als einige Bewohner*innen ausziehen mussten, trafen sie sich auch in ihren WGs oder an anderen Orten. Bei den Begegnungen ging es ständig um Gemeinsames und Unterschiedliches. Laura Otto wurde von den jungen Geflüchteten als weiße deutsche Frau gelesen, die sie manchmal wie ihre "große", manchmal wie ihre „kleine“ Schwester behandelten, sie waren sich altersnah und wurden allmählich vertrauter. Nähe zwischen der Forscherin und jungen geflüchteten Frauen entstand vor allem darüber, sich als Frauen zu verstehen und zu positionieren. Gleichzeitig reflektierten die jungen Frauen, dass sie und die Forscherin Frau-Sein keineswegs gleich erlebten und verwiesen auf Unterschiede untereinander. Trotz großer Unterschiede hinsichtlich ihrer Privilegien gehörte die sich als heterosexuelle Frau präsentierende Forscherin nicht zu dem Personenkreis, der Geflüchteten Vorschriften machte (Metje 2005). Durch den Sonderstatus einer weißen Person, die den Alltag teilt, wurde sie bei manchen zur Vertrauten, mit der sie über Ängste und Sorgen sprechen konnten. Insgesamt war es jedoch weder einfach, zu den jungen Frauen eine Beziehung aufzubauen, noch diese zu halten. Dafür gab es unterschiedliche Gründe, wie der folgende Absatz aufzeigt, indem er einen ersten Einblick in die Lebensumstände und Biographien der jungen geflüchteten Frauen gibt.

Nach ihrer Flucht erhielten sie kaum Geld von ihren Familien zur Unterstützung, weil ihr Weggang als Verrat aufgefasst wurde. Zudem berichteten sie davon, dass in Somalia vor allem die Frauen*, also ihre Mütter, das Geld verwalten würden und diese eher eine gute Beziehung zu

\footnotetext{
${ }^{4}$ „Wir" bezieht sich auf Laura Otto und Margrit E. Kaufmann. Wenn es um die Dynamiken und Betrachtungsweisen im Feld geht und wir uns auf Laura Ottos Feldforschung auf Malta beziehen, verwenden wir „ich“.
} 
ihren Söhnen hätten, die deshalb finanzielle Hilfe bekämen. Daraus resultierte, dass sie, deutlich öfter als die jungen Männer*, die ebenso Teil des Forschungsprojekts waren, zwei Jobs ausübten, in der Regel als Reinigungskräfte in Hotels, privaten Haushalten oder Restaurants. Die jungen Frauen beschrieben sich auf vielen Ebenen als benachteiligt und hatten fast alle einen abgelehnten Asylbescheid. ${ }^{5}$ Einige von ihnen befanden sich zum Forschungszeitpunkt im Prozess der Anfechtung der Entscheidung, andere wiederum hatten bereits die finale Ablehnung erhalten und befanden sich in noch unsichereren und prekäreren Situationen als die meisten der jungen männlichen* Geflüchteten, die in die Forschung einbezogen waren.

\subsection{Konstruktion von jungen Geflüchteten auf Malta}

Nach der Ankunft auf Malta wurden bis 2015 alle Menschen inhaftiert, die ohne ordnungsgemäße Dokumente eingereist waren. Da die Frauen, die an der Forschung teilnahmen, von den maltesischen Behördenmitarbeiter*innen als jung und potentiell minderjährig gelesen wurden, sollte während der Haft ihr chronologisches Alter bestimmt werden. Die als UAM Eingestuften wurden im Anschluss in einer staatlichen Betreuungseinrichtung untergebracht. Zum Forschungszeitpunkt betrieb die maltesische Regierung zwei solcher Heime. In einem wurden junge Frauen* und Männer* untergebracht, in dem anderen nur Männer*. Die als volljährig geltenden Frauen* wurden in einem Frauen*haus untergebracht.

Im Rahmen der Befragungen bei der Ankunft und den Altersfeststellungsverfahren wurden die jungen Frauen* wiederholt mit eurozentrischen Verständnissen von Kindheit und Adoleszenz konfrontiert: "We look at childhood too much from our local, Western perspective", reflektierte eine NGO-Mitarbeiterin (I, 07/15). ${ }^{6}$ Wenn die jungen Geflüchteten eine Biographieanzweiflung vermeiden wollten, musste ihre Erzählung zu vorherrschenden Auffassungen von Minderjährigen, Verletzlichkeit und einer Abwesenheit von Agency passen (Galli 2017). Gerade in sozialen Kontexten, in denen junge Menschen, besonders Mädchen*, schon früh verantwortlich handeln, verwischt - aus eurozentrischer Perspektive die Grenze zwischen Kindheit und Erwachsenenalter. Eurozentrische Vorstellungen von Jugend (SCEP 2012, 11) in Verbindung mit Geschlechterstereotypen (Sommers 2001, 3) und weiteren Zuordnungen wie Elternschaft und Herkunft beeinflussen den Status der Geflüchteten: Es ist also nicht nur die Frage nach Alter, die weitreichende Folgen für das Leben junger Geflüchteter hat (Otto 2020; Bhabha 2004); auch über die offiziellen Rechtskategorien hinaus werden sie permanent kategorisiert und sie positionieren sich stets neu in diesem Geflecht verschiedener Zuschreibungen und Erwartungen Dritter, offizieller und inoffizieller Rahmensetzungen sowie Wünsche an sich selbst.

Um solche multiplen, dynamischen Selbst- und Fremdzuordnungen aufzeigen zu können, verknüpfen wir die ethnographische Perspektive mit einer intersektionellen. Im folgenden Teil gehen wir auf den Ethnographie-Intersektionalitäts-Nexus ein und stellen unsere intersektionelle Leseweise ethnographischer Forschungsausschnitte vor.

\section{METHODOLOGISCHE VERBINDUNGEN VON ETHNOGRAPHIE UND INTERSEKTIONA- LITÄT}

Der ethnologischen Frauenforschung, die sich seit den 1950er-Jahren entwickelt hatte (Mead 1985) und die in die feministische Kulturanthropologie mündete, lag implizit ein intersektioneller Ansatz zugrunde. Der Begriff „Frau“ wurde früh als kulturelles, soziales Konstrukt, das mit weiteren sozialen Kategorien verbunden ist, betrachtet. Rückte zu Beginn eine ethnologische Frauenforschung in den Vordergrund, konzentrierte sich die Genderforschung in den 1980er- und 1990er-Jahren auf sozialkonstruk-

\footnotetext{
${ }^{5}$ Auf Malta wurden zum Forschungszeitpunkt drei Status vergeben: Flüchtlingsstatus, subsidiärer Schutz, sowie „Temporary Humanitarian Protection“. Wer keiner der drei Kategorien zugeordnet wurde, erhielt einen Ablehnungsbescheid, der auch „rejected“ heißt.

${ }^{6}$ I verweist auf Interviewzitate, TB bezeichnet Tagebucheinträge, IG steht für informelles Gespräch.
} 
tivistische, ethnomethodologische und dekonstruktive Ansätze (West/Zimmermann 1987; Reeves Sanday/Gallagher Goodenough 1990; Fenstermaker/West 2002). Sowohl den Black Feminist Studies, denen sich Crenshaw (1989) zuordnet, als auch der feministischen Ethnologie ging es darum, sich von universalistischen Verständnissen von „Geschlecht" und „Frau“ zu verabschieden (MacCormack/Strathern 1980; Stacey 1987; Brah 1996) und Unterschiede unter Frauen* zu fokussieren. Die Ethnologie trug dazu bei, die kulturelle Vielfalt von Gender, Begehrensausrichtungen, Ehe- und Familienformen im Rahmen jeweiliger Gesellschaftsverhältnisse in den Blick zu nehmen (Moore 1989, 1994). Gender wurde und wird hier als komplexe, ambivalente Unterscheidungskategorie im Zusammenhang mit anderen Differenzlinien und Diskriminierungsebenen erkundet.

\subsection{Intersektionalität als ethnographische For- schungshaltung}

Bereits die Rede von Soujourner Truth aus dem Jahre 1851 (vgl. Brezina 2004) zeigt, dass monokategorial ausgerichtete Perspektiven der Komplexität sozialer Ungleichheit und Unterdrückung, wie sie Schwarze Frauen* erleben, nicht gerecht werden. ${ }^{7}$ Die Idee einer "global sisterhood" wurde in den 1970er-Jahren vor allem von Schwarzen Feminist*innen in den USA kritisiert, die darauf verwiesen, dass sie nicht allein aufgrund ihres Geschlechts diskriminiert wurden. Um die Verwobenheit sozialer Ungleichheit betonen zu können, prägte die Juristin Kimberlé Crenshaw (1989, 1991) den Begriff der Intersektionalität. Sie legte damit sowohl ein Konzept der diskriminierungskritischen Analyse als auch einen Praxisbegriff vor, der Schutzmaßnahmen gegen Mehrfachdiskriminierung einfordert. Auch in aktuellen Ansätzen wie den Critical Diversity Studies (Kaufmann 2018, 2019) wird Ungleichheit bezüglich Gender und Heteronormativität als verwoben mit weiteren Ungleichheitsdimensionen untersucht.

Doch haben sich inzwischen viele methodologisch unterschiedlich positionierte Intersektionalitätsansätze entwickelt (McCall 2005; Anthias 2008; Degele/Winker 2009; Walgenbach 2012;
Biele Mefebue et al. 2021). Vor allem deutschsprachigen Ansätzen wird immer wieder attestiert, dass sie soziale Verhältnisse aus dem Blick verlieren, zu sehr auf Identitätspolitiken bezogen und insgesamt zu apolitisch seien (Soiland 2012; Erel et al. 2008). Dem wirken antikategoriale, praxeologisch orientierte ethnographische Ansätze entgegen, indem sie alltägliche Entstehungsprozesse von sozialer Ungleichheit thematisieren (Goel 2015, 2021; Binder/Hess 2011).

Statt im ethnographisch erhobenen Material vorgegebene Kategorien wiederzufinden oder Kategorien wie Schablonen auf das Material zu legen, möchten wir Prozesse des vielfältigen Umgangs mit wirkmächtigen Kategorien bei Fremd- und Selbstzuschreibungen nachvollziehbar machen. Um vorgefertigten Bildern über junge geflüchtete Frauen* zu entgehen, versuchen wir, wie es Goel (2015, 2021) als Ziel definiert, a priori gegebene Kategorien zu hinterfragen und stattdessen die dynamische Entstehung sozialer Positionierungen zu beleuchten. Dazu eignen sich ethnographische Forschungen, die in situ das doing von Selbst- und Fremdpositionierungen erfassen (Hirschauer 2014; Breidenstein et al. 2013; Hörning/Reuter 2004) und den von Goel geforderten Umgang mit Kategorien differenziert aufzeigen können. Dieses detaillierte Aufzeigen des Umgangs mit Kategorien und Klassifizierungen schlägt die Brücke zwischen Ethnographie, die im Kern induktiv vorgeht, und einer deduktiv vorgehenden Intersektionalität. Dabei werden Empirie und Theorie ständig in den "Austausch“ (Goel 2021, 3) gebracht. Geschlechterkonstruktionen werden entsprechend situativ und kontextbezogen erschlossen, indem Prozesse des doing, also feinteilige Interaktionen zwischen verschiedenen Akteur*innen, als gemeinsame, aber auch divergierende, ambivalente Differenzierungs- und Zuordnungsprozesse beobachtet und beschrieben werden (Goel 2020; Binder/Hess 2011).

Dass im Grenzregime Geschlecht und Alter wichtige Kategorien zur Unterscheidung von Personen und im Umgang mit diesen sind, verwundert nicht. Dies zeigte sich bereits bei der

\footnotetext{
7 Schwarz wird großgeschrieben, um zu verdeutlichen, dass es sich um ein konstruiertes Zuordnungsmuster handelt.
} 
Einteilung der jungen Geflüchteten nach der Ankunft und bei der Zuweisung in die Unterkünfte. In Anlehnung an Crenshaw (1989, 1991) gehen wir davon aus, dass gängige westliche Zuschreibungen - im Fall von Weiblichkeit beispielsweise mit passiv und verletzlich konnotiert - den multiplen Lebens- und Diskriminierungserfahrungen junger Schwarzer geflüchteter Frauen* nicht gerecht werden. Um aufzeigen zu können, wie sich jeweilige Zuschreibungen mit weiteren Selbstund Fremdpositionierungen verbinden und sich diese in durchmachteten Feldern, wie dem Grenzregime in Malta, konkret ausgestalten, nutzen wir Intersektionalität als Perspektive und Forschungshaltung (Goel 2020) zur gemeinsamen Lektüre ethnographischen Materials. Das Grenzregime ist dann nicht einfach deterministisch, es legt Positionierungen nicht (nur) fest, sondern ist ein Geflecht, in dem Zuschreibungen und Positionierungen mit dem und gegen den produzierten Diskurs verhandelt werden. Dieser Ansatz erlaubt es, den Umgang der Akteur*innen mit Selbst- und Fremdpositionierungen zu beleuchten (Binder/Hess 2011).

\subsection{Intersektionelle Leseweisen im rekursiven Forschungsdesign}

Eine intersektionelle Forschungshaltung und Leseweise ethnographischer Forschungsausschnitte kann Bestandteil eines für die Ethnographie charakteristischen, rekursiven Forschungsprozesses sein (Breidenstein et al. 2013). Dass wir dabei die jungen Frauen* eben nicht über essentialisierende Kategorien definieren möchten und dennoch von ihnen als Gruppe schreiben, verdeutlicht die Schwierigkeiten mit Benennungen, die sich intersektionellen Analysen stellen. Es entsteht eine Spannung zwischen Dynamiken, die sich zwischen reproduzierenden Kategorisierungen und antikategorialer Dekonstruktion abspielen (Goel 2021, 2), sowie zwischen inter- und intrakategorialen Verknüpfungen (Kaufmann 2019, 64 f.). Mit einem antikategorialen, dekonstruktiven Ansatz geht es uns in Anlehnung an McCall (2005) nicht darum, Kategorien und deren Wirksamkeit zu negieren oder zu ignorieren, sondern nach ihren Genealogien, Effekten und Transformationen zu fragen. Beim Lesen (ethnographischer) Forschungsmaterialien, die aus intersektioneller Perspektive machtvolle Konstruktionsmomente und -prozesse fokussieren, geht es uns um die Kritik an der Wahrnehmung von Menschen entlang gängiger Kategorien als Zuordnung zu hierarchisierten sozialen Gruppen. Zugleich geht es uns darum, Selbstzuordnungen wahrzunehmen, (gruppen-)spezifische Schwierigkeiten und geteilte Erfahrungen nicht zu vernachlässigen.

Die Herausforderung einer intersektionellen Leseweise besteht demnach darin, sich mit Zuschreibungsprozessen, mit denen junge Geflüchtete individuell und kollektiv konfrontiert sind, auseinanderzusetzen, ohne diese Zuschreibungen zu reproduzieren. Um die Wirkmächtigkeit sozial konstruierter Kategorien besser zu verstehen, versuchen wir, Kategorien als Markierungen eines umkämpften Feldes zu interpretieren (Brah 1996) und arbeiten den Umgang mit Kategorien induktiv aus dem Material heraus, um aufzeigen zu können, wie sie hervorgebracht, in Frage gestellt oder auch verworfen werden.

Da wir die Wissensproduktion als stets situiert verstehen, erachten wir es als relevant, die eigenen Positionen, Muster und Verständnisse zu reflektieren, beispielsweise durch die gemeinsame Leseweise des Forschungsmaterials. Wir haben die Erfahrung gemacht, dass gerade die Koautorinnenschaft, wie wir sie für diesen Beitrag praktizieren, eine Leseerfahrung ermöglicht, die Irritationen, Brüche und das Einfühlen in Beziehungsdynamiken zu entschlüsseln hilft. Die Person, die das Material erhoben hat, wird zudem einerseits produktiv herausgefordert, andererseits kollegial unterstützt, Alltagserlebnisse, Dynamiken und Prozesse sag- und schreibbar zu machen. Trotz des Austauschs und der Zusammenarbeit können wir gegenüber der Vieldeutigkeit des Materials in den folgenden empirisch orientierten Abschnitten nur einzelne mögliche Leseweisen aufzeigen.

\section{SITUATIVE AUSLEGUNGEN VON WEIB- LICHKEIT IM GRENZREGIME}

In diesem Teil widmen wir uns deutend verschiedenen Situationen, in denen die in der Fluchtsituation auf Malta aufeinander bezogenen Akteur*innen - junge Geflüchtete, sie Betreuende und Verwaltende sowie die Forscherin Formen von Weiblichkeit der jungen Frauen* mit Flucht_Migrationserfahrung besprechen und 
behandeln. Wir zeigen, wie darüber deren Weiblichkeit in Verbindung mit weiteren Zuschreibungen konstruiert wurde. Wir konzentrieren uns in diesem Beitrag auf Beschreibungen zu Erfahrungen und Erzählungen von Binti, Caasho, Tanaad, Muriyo, Nafiso und Aluna. Diese sechs jungen Frauen teilen die Erfahrung, dass sie in Somalia aufwuchsen und ohne Begleitung flohen. Über Äthiopien, den Sudan und Libyen kamen sie zwischen 2012 und 2013 auf Malta an, wo Laura Otto sie kennenlernte und Beziehungen zu ihnen aufbauen konnte. Binti, Muriyo und Aluna wurden als „minderjährig“ eingeteilt und lebten in der staatlichen Unterkunft für UAM. Caasho, Tanaad und Nafiso hingegen wurden als volljährig klassifiziert und lebten zunächst in einem Frauen*haus, bevor sie sukzessive mit Partnern* zusammenlebten oder mit anderen Frauen* Wohngemeinschaften gründeten.

\subsection{Wie Unsagbares sagbar machen? Erzählun- gen über Gewalt und Flucht}

Die Frage danach, ob und wie die jungen Frauen über ihre Fluchterfahrungen, die eng mit Gewalterfahrungen verbunden waren, gesprochen haben, ist von zentraler Bedeutung. Wir fragen danach, wie diese Erfahrungen Selbst- und Fremdpositionierungen beeinflussen. Im Fokus stehen die Erzählungen von Nafiso und Binti, die Mutterschaft und Gewalt in der Ehe und sexualisierte Gewalt gegen Frauen* auf der Flucht thematisieren.

Nafiso, die Malta 2012 nach mehrmonatiger Flucht erreichte, lernte Laura Otto im Sommer 2015 kennen. Zu dem Zeitpunkt war Nafiso in einer Beziehung mit Roodoo, einem jungen Mann aus Somalia, den Laura Otto gut kannte, weil er im Wohnheim für UAM lebte. Es war inm ein wichtiges Anliegen, seine Freundin vorzustellen und auch Nafiso stimmte einem Treffen sofort zu:

"Ich freute mich darüber und wir verabredeten uns auf einen Kaffee. Da Nafiso im Frauen*haus in Tal Gebel lebte, schlug sie vor, dass wir uns in einem der zahlreichen Strandcafés in Hal Balbi treffen könnten, denn der vielbesuchte Strandabschnitt lässt sich von Tal Gebel mit dem Bus in 20 Minuten erreichen. Es war gar nicht meine Absicht, mit ihr über ihre Gründe des Somalia-
Verlassens zu sprechen, aber sie erzählte von allein, dass sie unter ihrem gewaltvollen Ehemann litt und keinen anderen Weg sah, außer zu gehen. Nicht nur hat Nafiso ihren sozialen Kontext zurückgelassen, sondern auch ihr Baby: , I did not like that man. He was no good. He beat me. And me I have baby. Very young me I have baby. Now me Malta and the baby with the father. I go Malta because he beat too much'." (IG, 07/2015)

Nafiso machte in ihrer Erzählung deutlich, dass ihre genderspezifischen Gewalterfahrungen, wie die Frühverheiratung und die erlebte häusliche Gewalt, sie zur Flucht trieben. Sie erzählte, dass sie in Somalia in großer Angst lebte und oft schwere Verletzungen aus den Auseinandersetzungen mit dem Ehemann davontrug. Weinend berichtete sie, dass sie wiederholt über das Gehen nachgedacht hätte und abwog, ob sie die Gewalt durch ihren Ehemann weiterhin aushalten könne, um bei ihrem Sohn bleiben zu können. Trotz aller Verletzungen und Unsicherheiten sammelte sie die Kraft zu gehen und überstand die mehrmonatige Flucht nach Europa.

Nach der Ankunft auf Malta wurden Nafisos Erfahrungen für ihren Asylantrag relevant. Sie berichtete davon, dass ihr im Asylinterview sehr viele Fragen zu ihrer Biographie gestellt worden seien, die sie nicht immer beantworten konnte:

"In the interview, they ask too many questions. About everything. My life. My baby. And about other things. I did never hear about. They ask how many hours the driving between Mogadishu and other places takes. But me no idea. Because never I go to school, I never go away from my home much." (IG, 07/2015)

Nachdem Nafisos Asylgesuch abgelehnt wurde, lebte sie, wie viele andere geflüchtete Frauen*, mit einem doppelten Ablehnungsbescheid auf Malta. Die konkreten Gründe für die Ablehnung erfuhr sie nicht, doch vermutete sie, dass ihr trotz ihres jugendlichen Alters der Schutzstatus versagt wurde, weil sie ohne Schulbildung und zudem Mutter war. Im Interview, so reflektierte sie im Gespräch, sei sie immer wieder nach dem zurückgelassenen Sohn gefragt worden. Sie hatte den Eindruck, dass ihr nicht geglaubt wurde, weil sie es über sich brachte, ihr Kind beim gewaltvollen Vater zu lassen. 
Nafiso irritiert uns Deutende mit ihren Praktiken, ihrer Biographie und ihren Erzählungen. Passen sie doch so gar nicht zu unseren westlich geprägten Vorstellungen von Mutterschaft und Jung-Sein. Beim Deuten kreisten wir immer wieder um Vorurteile zu Teenie-Müttern, die eigentlich noch kein Kind haben sollten, Rabenmüttern, die der Fürsorgepflicht für ihr Kind nicht nachkommen, bzw. unserem Bild von einer guten Mutter (Vinken 2001) nicht entsprechen, und dem Verständnis für Nafisos Not und die persönliche Entscheidung sich selbst zu retten.

Ergriffen einige der jungen Frauen* aufgrund von Gewalterfahrungen am Herkunftsort die Flucht, so machten die meisten von ihnen weitere Gewalterfahrungen auf der Flucht. Denn mit dem Verlassen ihres sozialen Umfeldes ergaben sich neue Formen von Schutzlosigkeit, doch darüber wurde erstaunlich wenig gesprochen.

Im Laufe der Forschung ist aufgefallen, dass junge Frauen* in der Regel mit Laura Otto über andere junge Frauen* und ihre Fluchtgeschichten sprachen, doch nicht über sich selbst. Diese Gespräche kamen meistens bei gemeinsamen Aktivitäten, wie Strand- oder Marktbesuchen, Spaziergängen oder Ausflügen, zustande. Eine stabile Beziehung konnte zu Binti aufgebaut werden; der Kontakt zu ihr gestaltete sich recht unkompliziert, weil Binti immer wieder das Gespräch mit Laura Otto suchte:

„Beim Besuch eines Wochenmarkts, wo wir gemeinsam Lebensmittel für einen Kochabend im Wohnheim kaufen wollten, sprach sie mit mir über ihren Weg nach Malta. Sie käme aus der Nähe von Mogadischu und hätte ausschließlich kleinere Geschwister. Ihre Eltern seien schon lange getrennt. Sie hätte wiederholt das Thema ,Europe' bei beiden angesprochen, aber diese hätten sich sehr besorgt gezeigt. Sie kam aus ärmlichen Verhältnissen und die Familie war nur in der Lage, ihr Geld für den Weg bis in den Sudan zu geben. Binti berichtete mir, dass sie Somalia im Januar 2013 verlassen hatte, ohne die Eltern zu informieren, und am 29. März desselben Jahres in Malta angekommen sei. ,Really only three months, everybody says, Oh you were lucky, very lucky.' And I know I am. When I left Somalia, I did not believe that I survive, really, I was afraid. But now I am just very, very happy'." (IG und TB, 05/2013)

Sie erzählte, dass Libyen der schlimmste Ort gewesen sei, und berichtete von Mord, Folter und sexueller Gewalt. Binti selber, so meinte sie, hätte diese zum Glück nicht erfahren und sei dafür sehr dankbar. Sie berichtete aber, dass ihre Freundin Muriyo, die auch in dem Heim lebte, ganz andere Erfahrungen gemacht hätte:

"Muriyo, very bad. She needed one year from Somalia to Malta. Every time they catch her and send her back. In Libya, she was detention. And Libya they hate black women. They tell you come, come to the car. You have to go they have gun, you don't go they kill you. So, she was with magave [men in Libya who abuse female refugees], different magave. They beat, they punish, you are in house like prison, they keep women. And you know they do more bad things." (IG, 05/2013)

Der Kontakt zu Binti steht beispielhaft für das, was Laura Otto während der Forschung immer wieder erlebte: Es wurde kaum über sich, aber viel über andere gesprochen. Zwar benannte Binti ihre eigene Flucht, ging aber nicht weiter darauf ein. Über ihre Freundin Muriyo hingegen berichtete sie intime Details zu Gewalterfahrungen. Muriyo selbst hatte diese Erfahrungen jedoch in den gemeinsamen Gesprächen nie erwähnt. Bezüglich Bintis Erzählungen über körperliche Gewalt vermuten wir, dass sie auf diesem Wege indirekt über ihre eigenen Erfahrungen gesprochen haben könnte und das Erzählen über Gewalterfahrungen anderer eine Art Entlastungsfunktion gegenüber dem Sprechen über eigene schmerzhafte Erfahrungen hat. Das Betonen von Muriyos verletzlicher und verletzter Weiblichkeit wurde von Binti genutzt, so unsere Deutung, um überhaupt über Gewalt an Frauen* in Fluchtkontexten sprechen zu können. Nicht nur ist das Sprechen über eigene gewaltvolle Erfahrungen schambesetzt, bei vergewaltigten, missbrauchten Frauen* reaktivieren die Erinnerungen an diskriminierende Erfahrungen den Schmerz und die Pein. Binti wählte hier einen Weg, Erfahrungen und Ängste thematisieren zu können, ohne Angst haben zu müssen, selber (weiter) abgewertet zu werden. 
Die Erzählungen zeigen, dass die jungen Frauen zu Objekten gemacht wurden und immer wieder Opfer körperlicher und sexualisierter Gewalt waren. Sie selbst zeigten sich im Kontakt zur Forscherin als junge Frauen, die entgegen von Warnungen und Vorbehalten ihren eigenen Weg gingen. Während Nafiso über ihre Gewalterfahrungen sprach, wurde Binti gewissermaßen zum Sprachrohr für andere junge, weibliche Geflüchtete: Da sie sich selbst als unverletzt und stark zeigte, machte sie Unsagbares sagbar und fand einen Weg, über Gewalt gegen Frauen* zu sprechen. Zudem zeigen die Beispiele, dass sich die jungen Frauen gerade über (sexuelle) Gewalterfahrungen als weiblich positionierten, über die Rolle von Frauen* in der Gesellschaft nachdachten, innen durchaus widersprachen und nach eigenen weiblichen Lebensentwürfen suchten.

\subsection{Ungeschützt im Grenzregime: Ablehnung und Prekarität}

Die jungen Frauen, die an der Forschung teilnahmen, hatten in der Regel einen abgelehnten Asylbescheid, der ihren Zugang zu öffentlichen Versorgungsnetzwerken einschränkte. Zudem erhielten sie nach eigener Einschätzung deutlich weniger Unterstützung von ihrer Herkunftsfamilie als gleichaltrige Männer*, in die eher Hoffnung projiziert wurde. Im Ergebnis mussten sie sehr viel arbeiten und waren oft auf sich allein gestellt. Über den Schutzstatus bzw. die Abwesenheit dessen, wurde immer wieder gesprochen; oftmals erzählten die jungen Geflüchteten beim ersten Kontakt, wie ihr Asylinterview ausging. Auch der Status anderer war immer wieder Gesprächsthema.

Welche Auswirkungen die abgelehnten Bescheide auf das Wohlergehen und die Zukunft der jungen Geflüchteten hatten, wurde Laura Otto besonders bewusst, als sie im Wohnheim anwesend war, während Muriyo erfuhr, dass ihr Widerspruch erfolglos war und sie erneut einen negativen Bescheid erhalten hatte. In den Feldnotizen wurde Folgendes notiert:
„Heute kommt Muriyo völlig aufgelöst von ihrem Termin zurück. Man hat ihr gesagt, dass sie nun double reject hat. Es bedeutet für sie, dass sie in Zukunft kaum Zugang zur medizinischen Versorgung haben wird, nur das Überlebensnotwendige deckt der Staat ab. Zudem wird ihre Sozialhilfe auf 80 Euro im Monat begrenzt sein, womit ein selbstbestimmtes Leben unmöglich ist. Arbeiten kann sie ab heute nur noch illegal." (TB, 05/13)

Dass die Asylentscheide der jungen Frauen* oft abgelehnt wurden und diese sich in stark prekarisierten Situationen wiederfanden, war auch den jungen geflüchteten Männern bekannt, wie von Elais thematisiert wurde, der zusammen mit Muriyo im Wohnheim untergebracht war:

„Elais berichtet heute von seinem neuen Job bei LMAO. Er weiß, dass viele Frauen ein reject bekommen haben. Er geht davon aus, dass es für sie weniger schlimm sei als für Männer, die einen solchen Ablehnungsbescheid bekommen, und erklärt: The ladies if they have reject is more easy for them because they are beautiful and they can marry a man with subsidiary status and then they are safe. But the man he cannot find a woman here with good status. So that is a big problem. Always we help the Somali girls, and we can marry them if we have a good status, but they never help us.'“ (IG, 07/2015)

Elais problematisierte die doppelte Ablehnung von Frauen* nicht; vielmehr, so unsere Deutung, reproduzierte er hier, dass die Abhängigkeit der Frauen* von Männern* als normal gilt. Er argumentierte, dass doppelt abgelehnte Männer* es noch viel schwerer hätten und die jungen Frauen* einfach von ihrer Weiblichkeit Gebrauch machen könnten, um sich strategisch einen Mann* mit einem guten Status zu sichern. Elais erachtete eine pragmatische Lösung durch strategisches Einsetzen von Weiblichkeit als eine attraktive Möglichkeit, die ihm selbst verwehrt war, ohne sich Gedanken zu machen, was dies für die Frauen* beinhalten könnte.

Caasho war eine der jungen Frauen*, die gezielt geheiratet hatte, um sich einen besseren Status

\footnotetext{
${ }^{8}$ Local Migrant Aid Organisation (LMAO); anonymisierter Begriff für die staatliche Behörde, die u. a. für die Unterbringung geflüchteter Menschen zuständig ist.
} 
zu sichern. Sie machte gewissermaßen das, was Elais vorschlug, und heiratete Nuuroo aus diesem Grund. Zu ihr entwickelte sich im Laufe des Jahres 2015 ein enger Kontakt. Sie erzählte, dass sie nach ihrer Ankunft auf Malta als 19 Jahre alt eingestuft wurde und ihr Asylgesuch, ebenso wie das von Muriyo, zweimal abgelehnt wurde.

Diese pragmatischen Lösungen kollidierten immer wieder mit unseren westlich geprägten Vorstellungen von Geschlechtergerechtigkeit und (weiblicher) Selbstbestimmung. Im Gegensatz zu Elais deuteten wir Caashos Lage folglich nicht zunächst durch eine pragmatische Brille, sondern vielmehr durch eine kritische. Was für uns hier durchscheint, ist, dass Entscheidungen der institutionellen Grenzregimeakteur*innen - nämlich die Ablehnung junger Frauen* - ihre Abhängigkeit und Vulnerabilität in Fluchtsituationen verschärfen. Zudem wurden in Erzählungen wie der von Elais Frauen* auf Vorstellungen untergeordneter Weiblichkeit reduziert und damit stereotypisierte, dichotome Geschlechterbilder reproduziert. Gleichzeitig zeigte Caasho, dass sie durchaus reflektiert und aktiv war: Sie versuchte, ihre aufenthaltsrechtliche Situation zu verbessern. Hierzu verläuft unser Deutungsprozess entlang dem schmalen Grat zwischen Bewunderung der Agency der jungen Frauen* und gleichzeitigem Wunsch, zu betonen, in welche Situationen der fremdpositionierenden Abhängigkeit und Abwertung sie gerieten.

Geflüchtete Frauen* haben also offenbar, zumindest in Bezug auf die Statusvergabe, mehr Ablehnung erfahren als junge Männer*. In der Konsequenz befanden sie sich öfter in sehr prekären Situationen. Dass sie dadurch verletzlicher und schutzloser waren als junge Männer*, wurde erstaunlicherweise weder von den geflüchteten Männern*, noch von den Betreuenden und Verwaltenden artikuliert.

\subsection{In den Augen der anderen: zwischen Fremd- und Selbstbestimmung}

Prägend für die Situation der jungen Frauen* auf Malta waren auch die Erwartungen, die an sie gestellt wurden. Es waren sowohl junge geflüchtete Männer*, die bestimmte Erwartungen an ihr (weibliches) Verhalten hatten, als auch die Mitarbeiter*innen aus der staatlichen Unterkunft. Aber auch die jungen Frauen* selbst definierten und performierten nach ihrer Ankunft auf Malta ihre Weiblichkeit aus. Besonders deutlich wurden diese Dynamiken beim Thema Kleidungs- und Partner*innenwahl. Das Aussehen der jungen Frauen*, die Art wie sie sich anzogen und zurechtmachten, war ständig Thema:

„Caasho lud mich immer wieder ein, um mir beizubringen, somalische Sambuus (Teigtaschen) zu kochen. Als sie sich im April 2016 auffällig schminkte, ein enges Kleid anzog, eine paillettenbesetzte Handtasche aus dem Schrank holte, High Heels trug und mit ihrer Freundin Hamda, die sich ähnlich kleidete, ausgehen wollte, kam es zum Streit mit ihrem Ehemann Nuuroo. Während Caasho ihr Outfit und das Ausgehen als normal empfand -, I see every girl like this here. And I like. Me I like to look good"“ (TB, 04/2016) - wies Nuuroo sie lauthals vor allen Anwesenden zurecht: Sie sollte sich weniger auffällig kleiden und schminken. Das Ausprobieren von Caasho und ihr eigenes Empfinden dessen, was als "normal“" und in ihren eigenen Augen als schön galt, wurden hier auch durch die spezifischen Bedingungen ihrer Ehe begrenzt. Sie ging schließlich nicht mit ihrer Freundin aus, sondern blieb zuhause.

Es wurde auch viel darüber gesprochen, dass sich die jungen Frauen* in den Augen der geflüchteten Männer* anders verhielten als in Somalia. Auf das Ausprobieren junger Frauen* wurde mit Abwertung ihrer Weiblichkeit und Freiheitsbeschränkungen reagiert. So problematisierten die jungen geflüchteten Männer* immer wieder, dass sich die jungen Frauen* nach ihrer Ankunft auf Malta verändern würden:

"Den Tag heute verbringe ich mit Abdul, der mir erzählt, dass er 2013 in Aluna verliebt war. ,I was in love with Aluna really. 'Aber er sei auch sehr enttäuscht von ihr gewesen, denn: She made out with too many guys. When the Somali girls come to Europe they start enjoying themselves. I can tell you. They change their behavior and clothes and everything. But me I need a girl I can trust.“. (IG, 07/2015)

Er setzte das Ausprobieren der jungen Frauen* damit gleich, dass sie als Partnerin für ihn nicht mehr in Frage kommen. Er schien die Erwartung zu haben, dass sie sich weiter so verhalten und 
kleiden sollten, wie er es für somalische Frauen als normal interpretierte.

Dass Männer* im Kontext von Flucht_Migration immer wieder die Definitionsmacht darüber beanspruchten, was für die jungen Frauen* als normal und angemessen gilt, beobachtete Laura Otto auch als Dynamik zwischen Mitarbeitern und Bewohnerinnen im Heim: Als ich im Sommer 2013 mit einigen Bewohner*innen an den Strand fahren wollte, nahm mich Heimmitarbeiter Bernard bei Seite:

"Ich stelle mich ans Tor und warte, bis alle fertig sind. Bernard sucht mich auf und fängt an zu reden. Hin- und hergerissen zwischen meinen Wünschen, dass er einfach geht, aber ich auch mehr erfahren möchte, halte ich sein Gerede aus. Bernard meint, dass Tanaad gar keine Somalierin sei. Schließlich gehe ihr Rock nur bis zum Knöchel und nicht bis auf den Boden. , In Somalia, she would be dead. I think she is not even Somali. Some of them only wear the hijab to pretend that they are from Somalia. Usually, they dress like floor cleaners, really they clean when they walk.' Ich entgegne ihm: ,She speaks fluent Somali and is all the time with the Somalis, so I would not doubt it.' Er erwidert: ,Yes, believe me it happened before. I will keep an eye on her and report it.' Dem Mitarbeiter des Sicherheitsdienstes, der neu ist, erklärt er auch, dass die meisten schummeln und er es überprüfen wird." (TB, 06/2013)

Tanaad bekam nicht mit, welche wirkmächtigen Zuschreibungen an sie gemacht wurden. Bernard erklärte sie zur Asylmissbrauchstäterin. Hier wurde unter weiß Positionierten verhandelt, wie sich eine somalische Frau zu verhalten hat. Bernard bestätigte seine paternalistische Überlegenheit über die Schutzbefohlene und versuchte, die Forscherin zur Komplizin zu machen. Er sprach Tanaad ab, sich zu kleiden, wie sie es für schön und richtig hielt, also selbst darüber urteilen zu können, was somalisch ist, und behauptete, dass somalische Frauen* normalerweise wie Reinigungskräfte gekleidet seien, da ihr langer Rocksaum den Boden wische. Er konstatierte zudem, dass sie in Somalia tot sein würde, wenn sie sich nicht an die landestypische Kleidung hielte.
Die jungen Frauen erlebten viele Situationen, in denen es ihnen sehr erschwert wurde, selbstbestimmt zu handeln. Taten sie es dennoch, wurde ihr primär männliches* Umfeld skeptisch. Wir deuten diese Situationen so, dass sämtliche Reglementierungen - wie z. B. von der Kleider- bis hin zur Partner*wahl - in der Fluchtsituation auf Malta dynamisiert wurden. Vorstellungen von weißen und von Schwarzen Männern* über somalische Frauen* prägten den Alltag auf Malta. Die Stimmen und Wünsche der jungen Frauen wurden selten berücksichtigt. Oftmals gaben sie dem Druck von außen nach und sprachen nicht offen darüber, was das mit ihren Selbstwertgefühlen machte.

\section{ERGEBNISDISKUSSION}

Die in dieser Ausgabe fokussierte Thematik der Frage nach Erleben und Gestalten von Kindheit und/oder Adoleszenz in Flucht_Migrationskontexten konkretisierten wir am Beispiel der Perspektiven und Erfahrungen junger Frauen* auf Malta. Ihre adoleszente Weiblichkeit wurde in verschiedenen Weisen genutzt sowie mit Bedeutung aufgeladen und im Umgang mit vielfältigen Selbst- und Fremdpositionierungen produziert. Wir zeigten in Anlehnung an die Fragestellungen dieser Ausgabe, dass der Alltag und die sozialen Beziehungen der jungen Frauen* von diesen Dynamiken charakterisiert waren.

Aus dem Feldmaterial und unseren Deutungen lassen sich verschiedene Selbst- und Fremdpositionierungen ableiten, die wir zwar textuell fixieren, die jedoch situativ, dynamisch und unterschiedlich sind. Es handelt sich dabei um Prozesse des Weiblich-Werdens und WeiblichGemacht-Werdens (Goel 2021), die eng mit anderen Kategorisierungen verflochten sind, wobei intersektionelle Verbindungen von Fluchtstatus, Alter und Gender zentral sind. Die damit verbundenen Klassifizierungs- und Zuordnungsprozesse hatten vor Ort sowohl zur Folge, dass die jungen geflüchteten Frauen* in staatlichen Unterkünften wohnten, als auch, dass sie sich häufig in Situationen finanzieller Not befanden. Zudem waren sie vielfachen Be- und Abwertungen von außen ausgesetzt. So stellten Vertreter*innen des Grenzregimes bspw. ihre 
Biographien infrage und trugen damit zum prekären Status bei. Dass die jungen Frauen* als im heiratsfähigen Alter gelesen wurden, führte dazu, dass sie einerseits als potentielle Partner*innen erachtet wurden; andererseits galten sie, aufgrund ihres für junge Männer* immer wieder irritierenden Verhaltens auf Malta, für eine Ehe mit einem somalischen Mann* als nicht mehr geeignet.

Abschließend führen wir die mittels einer an Intersektionalität orientierten Perspektive gehobenen Selbst- und Fremdpositionierungen junger geflüchteter Frauen zusammen. Gemäß dem Ziel, ihre Erfahrungen und Situation in einem Grenzregime am Beispiel Malta sichtbar(er) zu machen, wurden drei Narrative und Umgangsweisen der Selbst- und Fremdpositionierung im Umgang mit Weiblichkeit betont: Sprechen über Flucht und Gewalt, Umgang mit einem abgelehnten Schutzstatus sowie Erwartungshaltungen jungen geflüchteten Frauen* gegenüber.

Das (Nicht-)Sprechen über Gewalterfahrungen und die Flucht nach Malta zeigte, dass diejenigen, die über die Verletzungen anderer junger Frauen* sprachen, sich selbst als stark und migrationserfolgreich darstellten. Während das Sprechen über das Leid und die Gewalterfahrungen Dritter dazu beitrug, dass eine Erzählerin sich, von eigenen Erfahrungen entlastend, als starke junge Frau positionieren konnte, wurden diejenigen, über die gesprochen wurde, als verletzte und verletzliche Frauen* positioniert, ohne dass sie selbst davon wussten. Keine von ihnen musste dadurch über schambesetzte eigene Verletzungen sprechen. Anders verhält es sich in Fällen, in denen die jungen Frauen über eigene Erfahrungen sprachen, denn sie thematisierten dann nicht nur offen ihre Verletzlichkeit, sondern positionierten sich auch als junge Frauen, die bewusste Entscheidungen treffen konnten und die die Stärke aufbrachten, nach Europa zu fliehen. Von anderen hatten sie dafür nicht zwangsläufig Anerkennung erfahren.

In Bezug auf den Umgang mit dem (nicht erteilten) Schutzstatus positionierten sich die jungen Frauen immer wieder als Unwissende, die das Asylsystem in Malta aufgrund fehlender Bildung nicht durchschauten. Sie fühlten sich diesbezüglich gegenüber ihren männlichen* Gleichaltrigen benachteiligt. Das Gefühl, aus dem Rahmen zu fallen, wurde von außen verstärkt, indem ihre Kleidung für unpassend gehalten wurde, sie die Fragen im Interview nicht hinlänglich beantworten konnten, ihre Erfahrungsberichte als Lügen denunziert und sie, des Asylmissbrauchs verdächtigt, für schutzunwürdig erklärt wurden. Von männlichen* Geflüchteten wurde ihnen zugeschrieben, dass sie mit ihrer Weiblichkeit pragmatisch umgehen und sie strategisch einsetzen sollten, um einen Mann* mit Schutzstatus an sich zu binden. Die Interessen und Präferenzen der jungen Frauen spielten in diesen Erzählungen keine Rolle; stattdessen wurde ihre Abhängigkeit von Männern* normalisiert und durch das Grenzregime erweitert.

Entlang von Kleidungspraktiken und Partner*wahl wurden die heterogenen Selbst- und Fremdpositionierungen, die durch Erwartungen Dritter gefördert wurden, besonders gut sichtbar. Wenn die jungen Frauen romantische Beziehungen zu verschiedenen Männern* führten, wurden sie für einen somalischen Mann* als nicht mehr geeignet abgewertet. Während die jungen Frauen ein Interesse daran hatten, sich nach ihren eigenen Maßstäben schön zu finden und neue Modestile auszuprobieren, wurde ihnen von ihren männlichen* Gleichaltrigen verdeutlicht, dass sie sich so kleiden sollten, wie es Männer* für eine somalische Frau* als angemessen erachteten.

Um diese Dynamiken erkennen und beschreiben zu können, arbeiteten wir entlang des Ethnographie-Intersektionalität-Nexus. Zu der Frage, welche empirischen Zugänge sich eignen, um sich forschend Erfahrungen und Perspektiven junger geflüchteter Menschen anzunähern, die das Thema dieses Themenhefts darstellen, schlagen wir vor, Ethnographie und Intersektionalität zu verbinden. Eine an Intersektionalität orientierte Leseweise mit einem Verständnis von Selbstund Fremdpositionierungen als dynamische, interaktive Praktiken verdeutlicht, dass Gender, Fluchtstatus und Alter nicht nur wirkmächtige Kategorien für junge weiblich gelesene Geflüchtete sind. Sie bietet den analytischen Vorteil, zu zeigen, wie diese Selbst- und Fremdpositionierungen in durchmachteten Feldern konkret ausgestaltet werden. Um über junge Frauen* überhaupt schreiben und sprechen zu können, 
war für uns das gemeinsame Deuten und Lesen ethnographischen Materials emotional stützend und analytisch wertvoll. Dieser empirisch-analytische Zugang eignet sich unseres Erachtens, um den Umgang mit Weiblichkeit und daraus resultierende Selbst- und Fremdpositionierungen der jungen Frauen* erkunden zu können. Zu einer an Intersektionalität orientierten Analyse gehört es auch, das Nicht-Gesagte mit zu bedenken (Goel 2021); auch darin finden sich wichtige Aspekte der Alltagssituation auf Malta. In den Erzählungen über die jungen Frauen*, vor allem, wenn Männer* sprachen, war es fast nie Thema, wie es den jungen Frauen* geht: Wie sie das Erlebte verarbeiten, wie sie ihre Zukunft sehen, was sie möchten - das waren Themen und Fragen, die kaum verhandelt wurden. Im Kontakt mit der Forscherin jedoch sprachen die jungen Frauen genau das an: Sie hofften darauf, dass sie Zugang zu Bildung finden würden, viele wollten Ärztin oder Lehrerin werden. Sie hatten als Ziel, ein selbstbestimmtes Leben führen zu können, selbst einen Partner* zu finden. Sie betonten immer wieder, dass ihr Jung-Sein auch die Chance berge, ihre Ziele noch erreichen zu können.

Sicher ist unser Zugang begrenzt durch unsere eigenen Positionierungen, unsere Erfahrungen und das, was wir als normal empfinden. Selbstund Fremdpositionierungen zu Weiblichkeit in Grenzregimen sind multipel und konnten nur ansatzweise beschrieben werden. Spezifische Erzählungen und Lebenssituationen von geflüchteten Mädchen* und Frauen*, die es bis nach Europa schafften, haben in diesem Artikel Aufmerksamkeit erfahren und finden in zukünftigen Forschungen hoffentlich weiter ausdifferenziert Gehör.

\section{LITERATUR}

Anthias, Floya (2008): Thinking through the lens of translocational positionality: an intersectionality frame for understanding identity and belonging. In: Translocations - Migration and Social Change, 4 (1), 5-20.

Bhabha, Jacqueline (2004): From Citizen to Migrant: The Scope of Child Statelessness in the Twenty-First Century. In: Dies. (Hrsg.): Children Without a State: A Global Human Rights Challenge. Cambridge: MIT Press, 1-39.
Biele Mefebue, Astrid/Bührmann, Andrea/Grenz, Sabine (2021): Handbuch Intersektionalität. Wiesbaden: Springer.

Binder, Beate/Hess, Sabine (2011): Intersektionalität aus der Perspektive der Europäischen Ethnologie. In: Hess, Sabine, Langreiter, Nikola, Timm, Elisabeth (Hrsg.): Intersektionalität Revisited. Empirische, theoretische und methodische Erkundungen. Bielefeld: transcript, 15-52.

Brah, Avtar (1996): Cartographies of Diaspora: Contesting Identities (Gender, Race, Ethnicity). London: Routledge.

Breidenstein, Georg/Hirschauer, Stefan/Kalthoff, Herbert/Nieswand, Boris (2013): Ethnografie. Die Praxis der Feldforschung. Konstanz: UVK Verlagsgesellschaft.

Brezina, Corona (2004): Soujourner Truth's ,Ain't I a Woman?' Speech. A Primary Source Investigation. New York: Rosen Publishing, 25-33.

Çalışkann, Selmin (2018): Warum Frauen fliehen: Fluchtursachen, Fluchtbedingungen und politische Perspektiven. In: Heinrich Böll Stiftung (Hrsg.): Frauen und Flucht: Vulnerabilität - Empowerment-Teilhabe. Dossier. Online verfügbar unter: https://heimatkunde.boell.de/sites/default/ files/frauen_und_flucht_17_04_18_1.pdf

(18.08.2020).

Callamard, Agnès (2002): Refugee Women: A Gendered and Political Analysis of the Refugee Experience. Wiesbaden: Springer.

Crenshaw, Kimberlé (1989): Demarginalizing the Intersection of Race and Sex: A Black Feminist Critique of Antidiscrimination Doctrine. In: The University of Chicago Legal Forum, 1989 (8), 139-167.

Crenshaw, Kimberlé (1991): Mapping the Margins: Intersectionality, Identity Politics, and Violence against Women of Color. In: Stanford Law Review, 43 (6), 1241-1299.

Deacon, Zermarie/Sullivan, Cris (2009): Responding to Complex and Gendered Needs of Refugee Women. In: Affilia - Journal of Women and Social Work, 24 (3), 272-284.

Degele, Nina/Winker, Gabriele (2009): Intersektionalität. Zur Analyse sozialer Ungleichheit. Bielefeld: transcript. 
Erel, Umut/Haritaworn, Jin/Rodríguez, Encarnación Gutiérrez/Klesse, Christian (2008): On the Depoliticisation of Intersectionality Talk: Conceptualising Multiple Oppressions in Critical Sexuality Studies. In: Kuntsman, Adi/Esperanza, Miyake (Hrsg.): Out of Place: Interrogating Silences in Queerness/Raciality. New York: Raw Nerve Book, 265-292.

Fenstermaker, Sarah/West, Candance (Hrsg.) (2002): Doing Gender, Doing Difference. New York/London: Routledge.

Galli, Chiara (2017): A rite of reverse passage: the construction of youth migration in the US asylum process. In: Ethnic and Racial Studies, 40 (7), 1-21.

Gerard, Alison/Pickering, Sharon (2013): Gender, Securitization and Transit: Refugee Women and the Journey to the EU. In: Journal of Refugee Studies, 27 (3), 338-359.

Goel, Urmila (2015): From Methodology to Contextualisation. The Politics and Epistemology of Intersectionality. In: Presses de Sciences, 2 (58), 25-38.

Goel, Urmila (2020): Das Indernet. Eine rassismuskritische Internet-Ethnografie. Bielefeld: transcript.

Goel, Urmila (2021): Intersektional Forschen kontextspezifisch, offen, selbst-reflexiv. In: Biele Mefebue, Astrid/Bührmann Andrea/Grenz Sabine (Hrsg.): Handbuch Intersektionalitätsforschung. Wiesbaden: Springer VS. https://doi.org/10.1007/978-3-658-266134_16-1

Hanevinkel, Vera (2018): Frauen in der Migration: Ein Überblick in Zahlen. Online verfügbar unter: https://www.bpb.de/gesellschaft/migration/kurzdossiers/280217/ein-ueberblick-in-zahlen (15.02.2021).

Hess, Sabine/Tsianos, Vassilis (2010): Ethnographische Grenzregimeanalyse. In: Hess, Sabine/Kasparek, Bernd (Hrsg.): Grenzregime. Diskurse, Praktiken, Institutionen in Europa. Berlin: Assoziation A, 243-264.

Hirschauer, Stefan (2014): Un/doing Difference. Die Kontingenz sozialer Zugehörigkeiten. In: Zeitschrift für Soziologie, 43 (3), 170-191
Hörning, Karl/Reuter, Julia (2004): Doing Culture. Neue Positionen zum Verhältnis von Kultur und sozialer Praxis. Bielefeld: transcript.

Huxel, Karin (2014): Männlichkeit, Ethnizität und Jugend: Präsentationen von Zugehörigkeit im Feld Schule. Wiesbaden: Springer VS.

Kaufmann, Margrit E. (2018): Mind the Gaps. Diversity als spannungsgeladenes Zeitgeist-Dispositiv. In: Florin, Moritz/Gutsche, Victoria/Krenz, Natalie (Hrsg.): Diversität historisch. Repräsentationen und Praktiken gesellschaftlicher Differenzierung im Wandel. Bielefeld: transcript, 212231.

Kaufmann, Margrit E. (2019): Intersectionality Matters! Zur Bedeutung intersektioneller kritischer Diversity Studies für die Hochschulpraxis. In: Darowska, Lucyna (Hrsg.): Diversity an der Universität. Bielefeld: transcript, 53-83.

Kholi, Ravi (2007): Social Work with Unaccompanied Asylum Seeking Children. Bedford: Red Globe Press.

Kholi, Ravi/Kaukko, Mervi (2018): The Management of Time and Waiting by Unaccompanied Asylum-Seeking Girls in Finland. In: Journal of Refugee Studies, 31 (4), 488-506.

Lutz, Helma/Amelina, Anna (2021): Gender in Migration Studies: From Feminist Legacies to Intersectional, Post- and Decolonial Prospects. In: Journal of Migration Research, 1 (1), 55-73.

MacCormack, Carol/Strathern, Marilyn (1980): Nature, Culture and Gender. Cambridge: Cambridge University Press.

McCall, Leslie (2005): The Complexity of Intersectionality. In: Signs: Journal of Women in Culture and Society, 30 (3), 1771-1800.

Mead, Margaret (1985): Mann und Weib. Das Verhältnis der Geschlechter in einer sich wandelnden Welt. Reinbek: Rowohlt.

Metje, Ute (2005): Zuhause im Übergang. Mädchen und junge Frauen am Hamburger Hauptbahnhof. Frankfurt am Main: Campus.

Moore, Henrietta L. (1989): Feminism and Anthropology. Cambridge: Polity Press.

Moore, Henrietta L. (1994): A Passion for Difference. Essays in Anthropology and Gender. Cambridge: Polity Press. 
Opitz, Sven (2011): Grenzregime. In: FuchsHeinritz, Werner/Stäheli, Urs (Hrsg.): Lexikon zur Soziologie. 5. überarbeitete Auflage. Wiesbaden: VS Verlag, 259.

Otto, Laura (2020): Junge Geflüchtete an der Grenze. Eine Ethnographie zu Altersaushandlungen. Frankfurt am Main/New York: Campus.

Otto, Laura/Kaufmann, Margrit E. (2018): „Minderjährig“, „männlich“, „stark“? - Komplexe Bedeutungsaushandlungen der Selbst- und Fremdzuschreibung junger Geflüchteter in Malta. In: GENDER, 2, 63-78.

Otto, Laura/Kaufmann, Margrit E. (2020): When generalized assumptions of young refugees don't hold. Rethinking ascriptions and subjectivations through an intersectional lens. In: Journal of Ethnic \& Cultural Diversity in Social Work, 29(13), 136-153.

Pickering, Sharon (2011): Women, Borders and Violence. Current Issues in Asylum, Forced Migration and Trafficking. Wiesbaden: Springer.

Pittaway, Eileen/Bartolomei, Linda (2001): Refugees, Race, and Gender: The Multiple Discrimination against Refugee Women. In: Refuge, 19 (6), 21-32.

Reeves Sanday, Peggy/Gallagher Goodenough, Ruth (1990): Beyond the Second Sex. New Directions in the Anthropology of Gender. Philadelphia: University of Pennsylvania Press.

SCEP (Separated Children in Europe Programme) (2012): Positionspapier zur Altersfestsetzung bei unbegleiteten Minderjährigen in Europa. Online verfügbar unter: http://www.refworld.org/cgi-bin/texis/vtx/ rwmain/opendocpdf.pdf?reldoc=y\&docid $=$ $\underline{563714674}$ (09.01.2021).

Scheibelhofer, Paul (2019): Prekarisierte Männlichkeit im Fluchtkontext. Erfahrungen eines jungen Mannes zwischen Syrien und Österreich. In: Böttcher, Alexander/Hill, Marc/Rotter, Anita/Schacht, Frauke/Wolf, Maria A./Yildiz, Erol (Hrsg.): Migration bewegt und bildet. Kontrapunktische Betrachtungen. Innsbruck: Innsbruck University Press.
Sirriyeh, Ala (2013): Inhabiting Borders, Routes Home. Youth, Gender, Asylum. Farnham: Ashgate.

Soiland, Tove (2012): Die Verhältnisse gingen und die Kategorien kamen. Intersectionality oder Vom Unbehagen an der amerikanischen Theorie. Online verfügbar unter: http://portal-intersektionalitaet.de/theoriebildung/ueberblickstexte/soiland/ (06.02.2021).

Sommers, Marc (2001): Youth - Care \& Protection of Children in Emergencies: A Field Guide. Online verfügbar unter: https://www.researchgate.net/publication/44837549_Y-

outh_care_and_protection_of_children_in_emer gencies_a_field_guide $(06.02 .2021)$.

Spijkerboer, Thomas (2000): Gender and Refugee Status. London: Routledge.

Spindler, Susanne (2006): Corpus Delicti. Männlichkeit, Rassismus und Kriminalisierung im Alltag von jugendlichen Migranten. Münster: Unrast Verlag.

Stacey, Jackie (1987): Desparatly Seeking Difference. In: Screen, 28(1), 48-61.

Tunç, Michael (2018): Väterforschung und Väterarbeit in der Migrationsgesellschaft. Rassismuskritische und intersektionelle Perspektiven. Wiesbaden: Springer VS.

Vervliet, Marianne/De Mol, Jan/Broekaert, Jan/Derluyn, Ilse (2013): 'That I Live, that's Because of Her': Intersectionality as Framework for Unaccompanied Refugee Mothers. In: British Journal of Social Work, 44 (7), 2023-2041.

Vinken, Barbara (2001): Die deutsche Mutter. Der lange Schatten eines Mythos. München/Zürich: Piper.

Walgenbach, Katharina (2012): Intersektionalität - eine Einführung. Online verfügbar unter: http://portal-intersektionalitaet.de/theoriebildung/schluesseltexte/walgenbach-einfuehrung/ (04.02.2021).

West, Candace/Zimmermann, Don H. (1987): Doing Gender. In: Gender and Society, 1/2, 125151. 


\section{Zu den Autor*innen}

Laura Otto ist wissenschaftliche Mitarbeiterin am Institut für Kulturanthropologie und Europäische Ethnologie der Goethe-Universität in Frankfurt am Main. Sie promovierte mit einer ethnographischen Studie zu Altersaushandlungen an der europäischen Außengrenze. Zu ihren Forschungsinteressen gehören Fluchtmigrationsbewegungen junger Menschen, Governance und Grenzregimeforschung.

Margrit E. Kaufmann ist Senior Researcher am Institut für Ethnologie und Kulturwissenschaft an der Universität Bremen, wo sie zudem wissenschaftliche Expertin für Diversity ist. Zu ihren Schwerpunkten in Lehre und Forschung gehören intersektionelle, kritische Diversity Studies, ebenso wie Gender/Queer/Postcolonial Studies sowie Studien zu Rassismus und institutioneller Diskriminierung.

\section{Kontakt}

Dr. Laura Otto

Goethe-Universität Frankfurt am Main

Institut für Kulturanthropologie und Europäische Ethnologie

Norbert-Wollheim-Platz 1

60323 Frankfurt

E-Mail: otto@em.uni-frankfurt.de

URL: https://www.uni-frankfurt.de/77690794/Dr_L_aura_Otto

Dr. Margrit E. Kaufmann

Universität Bremen

Institut für Ethnologie und Kulturwissenschaft

Enrique-Schmidt-Str. 7

28359 Bremen

E-Mail:mkaufm@uni-bremen.de

URL: https://www.uni-bremen.de/kultur/personen/lektorinnen/margrit-e-kaufmann 